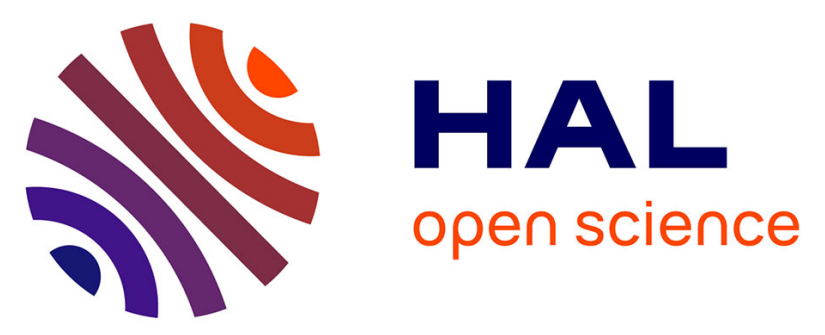

\title{
Adaptive Multipoint Model Order Reduction Scheme for Large- Scale Inductive PEEC Circuits
}

Trung-Son Nguyen, Tung Le Duc, Thanh-Son Tran, Jean-Michel Guichon, Olivier Chadebec, Gérard Meunier

\section{- To cite this version:}

Trung-Son Nguyen, Tung Le Duc, Thanh-Son Tran, Jean-Michel Guichon, Olivier Chadebec, et al.. Adaptive Multipoint Model Order Reduction Scheme for Large- Scale Inductive PEEC Circuits. IEEE Transactions on Electromagnetic Compatibility, 2017, 59 (4), pp.1143-1151. 10.1109/TEMC.2016.2641451 . hal-02277797

\section{HAL Id: hal-02277797 \\ https://hal.science/hal-02277797}

Submitted on 19 Oct 2020

HAL is a multi-disciplinary open access archive for the deposit and dissemination of scientific research documents, whether they are published or not. The documents may come from teaching and research institutions in France or abroad, or from public or private research centers.
L'archive ouverte pluridisciplinaire HAL, est destinée au dépôt et à la diffusion de documents scientifiques de niveau recherche, publiés ou non, émanant des établissements d'enseignement et de recherche français ou étrangers, des laboratoires publics ou privés. 


\title{
Adaptive Multipoint Model Order Reduction Scheme for Large-Scale Inductive PEEC Circuits
}

\author{
Trung-Son Nguyen, Tung Le Duc, Thanh-Son Tran, Jean-Michel Guichon, \\ Olivier Chadebec, and Gérard Meunier
}

\begin{abstract}
The model order reduction techniques based on the multipoint projection Krylov methods have become the methods of choice to generate large macromodels of multiport RLC circuits. A well-known difficulty with such methods lies in the need for clever point selection to attain model compactness and accuracy. In this paper, we present an automatic methodology for optimizing sample point selection. This method is general, suitable for circuits obtained by the partial element equivalent circuit method coupled with an adaptive multilevel fast multipole method. Our algorithm has been validated on an industrial example to demonstrate the accuracy and robustness of the approach.
\end{abstract}

Index Terms-Adaptive multilevel fast multipole method (AMLFMM), model order reduction (MOR), moment-matching method, partial element equivalent circuit (PEEC method), RLC.

\section{INTRODUCTION}

$\mathbf{P}$ ARTIAL ELEMENT EQUIVALENT CIRCUIT (PEEC) approach [1] is known to be very suitable for the modeling of combined circuit and electromagnetic field problems. For low-frequency applications, capacitive effects can be neglected and the method can be restricted to its inductive formulation [2], [3]. With such approach, arbitrarily shaped 3-D conducting devices can be represented by equivalent circuits combining resistors, partial/mutual inductances, and current and/or voltage sources. The circuit-based model of electromagnetic devices allows its interface with SPICE-like circuit solvers. This approach is known to be very efficient for the electromagnetic modeling of power electronic modules for instance [2], [3] and finely model can be coupled with the external electrical circuit.

The increment of number of conductor elements (directly proportional with the complexity of the devices) causes an

M anuscript received Novem ber 9, 2016; revised Novem ber 29, 2016; accepted D ecember 7, 2016. This research is funded by Vietnam $\mathrm{N}$ ational Foundation for Science and Technology Development (NAFO STED) under grant num ber 102.01-2014.39. (Corresponding author: Dr. Tung Le Duc.)

T.-S. Nguyen is with the Vietnam Fire and Rescue Police Department, Hanoi 10000, Vietnam (e-m ail: sonngtrung@ gm ail.com ).

T. Le Duc is with the Department of Electric Power Systems, Hanoi University of Science and Technology School of Electrical E ngineering, H anoi 10000, Vietnam (e-m ail: tung.leduc1@hust.edu.vn).

T.-S. Tran is with the Faculty of Electrical Engineering, Electric Power University, $\mathrm{H}$ anoi 100000 , Vietnam (e-m ail: sontt@epu.edu.vn).

J.-M. Guichon, O. Chadebec, and G . Meunier are with the G2ELA B, G renoble 38000, France (e-mail: Jean-Michel.Guichon@g2elab.grenoble-inp.fr; Olivier. Chadebec@g2elab.grenoble-inp.fr; gerard.meunier@g2elab.grenoble-inp.fr). explosion in memory by the number of terms in the full PEEC matrix. In order to save the memory and the computation time, we treat the interactions between far-away basis functions using the fast multipole method (FMM) [2]. The corresponding matrix elements do not need to be explicitly stored, result in a significant reduction in required memory. By using FMM, the resulted model is available only in the frequency domain and the link with a conventional circuit representation is lost. To be included in time-domain simulation of circuit-solvers, this model needs to be replaced by a reduced-order model, which is much smaller but would produce a very good approximation of input-output behavior of the original device.

This problem can be done in two steps. The first step is the reduction order of frequency model returned by PEEC. We then obtained a simpler and lighter reduced-order model. In the second step, this reduced model is synthesized into a circuit model in netlist representation (RLC elements), which can be integrated in a circuit-solver [4]. The pure RLC model is then simple enough to ensure the convergence of the resolution and accurate enough to return reliable results. The second step is presented in our recent work [5]. In this paper, we investigate the first step (reduction of frequency model): the model order reduction (MOR).

MOR methods for linear model reduction have greatly evolved and can be broadly characterized into two types: those based on subspace generation and projection (or momentmatching method), and those based on balancing techniques [6]. The balancing techniques can generate models with provable general error-bound [7], on the contrary, global error is unknown by projection methods [8]. However, the balancing techniques rely upon dense matrix computations, which seem unable to deal with large scale PEEC circuit compressed by FMM. In this case, due to a matrix compression algorithm, only projection method is suitable.

The projection method has led to construct the projection space from a rational or multipoint Krylov subspace [9]. The multipoint approximants are usually more expensive to construct than single point interpolation as PRIMA [10], but they tend to have better quality models for given effort [7]. Multipoint-based approaches [11]-[15] have recently gathered renewed attention due to their robustness, reliability.

Although very appealing, a major drawback of such methods in practical implementation is: how many interpolation points to choose, and how to place them. For large-scale systems, the computation cost of Krylov subspace at each expansion point 
is expensive, which makes the choice of these points is very important.

Until now, this area of research has barely been touched upon and the problems above still remain open. An early method called Complex Frequency Hopping (CFH) is proposed in [16] and [17] to illustrate a principle of choosing multiple expansion points of the transfer function. By using a binary search algorithm, the expansion points are chosen with respect to the common poles contained in both circles of the neighboring expansion points. However, the choice of expansion points is not optimal, which can lead to a large number of expansion points. The ARMS technique in [12] proposed a fully automated procedure to choose expansion point, which is based on maximizing the subspace spawned by the sampling set in order to obtain a good approximation of the dominant subspace in the orthonormalization step (construction of Krylov subspace). However, this approach is not specifically directed to point placement but to choose the best future point in a set of initial point.

Obviously, strategies include random selection of points or uniform grinding of the domain. Both of these can be shown as uninformed algorithms about either the structure or the behavior of the system, and as such, they may not be optimal. Resampling plans have been proposed in [13] to provide guidance for future sample point placement in linear MOR, based on variance measurements of the reduced transfer function. However, it requires the evaluation of several reduced models, which may be expensive, and, in addition, seem awkward to extend to multidimensional cases.

Intuitively, an efficient point selection algorithm should place new points, where the error or model uncertainty is large without evaluating the detailed model, which is expensive. There is no general answer to this question but we know some points could have large error: $f_{\min }, f_{\max }$, poles of the models (peak point in frequency response) [18]. Unfortunately, these poles are not known a priori. The work in [19] for SISO systems and a MIMO version [20] shows how to compute these points by an iterative procedure and the algorithm is fast convergence. It aims at automatically finding the optimal $\|\mathrm{H}\|_{2}$-norm model for a fixed $q$ th order, based on a refinement of the interpolation points. However, the algorithm needs to solve the original system at $r$-points at each refinement iteration, which makes the procedure expensive. The authors in [21], [22] proposed a cumulative reduction scheme (CURE framework), which offers more flexibility version of IRKA [16] without loss of precision. In this method, the authors reuse the previous constructed Krylov space to reduce computation cost. A recent work in [11] presented a fully adaptive scheme for Krylov-based MOR. Although, the proposed approach provides an interesting way of adaptive choosing moment $(q)$, the computation time is rather high because at each expansion point we need to compute the original model and the reduced model. The author in [23] also proposed a greedy multipoint MOR technique applied for second-order systems and a "goal-oriented" error estimator different than the system's impedance. Recently, there are many research related to MOR methods for finite element method [33], [34] but it is difficult to apply for our research because of the fully dense matrix in PEEC method (FEM matrix is sparse).
The multipoint MOR method for delayed PEEC circuits including the capacitive effects [14] and parameterized MOR [35] have been developed recently. In our research, the PEEC formulation is restricted on the inductive effects but it can be extended to general case as in [14] because the fully dense and sparse matrices are all treated in our study. An interesting approach for adaptive choice of expansion point is presented in [35] but the validation process of the method needs the computation of original model. In our approach, the exact computation of original model is impossible because of FMM algorithm. We also avoid the high computation cost of full model and focused on memory and time saving for large-scale application.

In this paper, we will propose a novel methodology for automated selection of optimal expansion points for first-order systems. The procedure not only shares some ideas in [11] and [19] but also exploits some properties of Krylov subspace and thick-restart techniques [24] to reduce the computation cost as much as possible. The originality of this study is its formulation coupled with the FMM method and a novel adaptive algorithm for selecting expansion points automatically in regard to minimize computation cost.

The paper is organized as follows: in Section II, the back ground state space equation of PEEC method is presented. In Section III, we discuss the implementation of our algorithm in presence of FMM and an adaptive point selection algorithm is introduced in Section IV. For the Section V, we show a computational experiment to illustrate the advantage of proposed approach and give several conclusions in Section VI.

\section{PEEC RLMC CIRCUIT EQUATIONS}

Considering a RLMC circuit (resistor-R, inductance-L, mutual inductance- $-\mathrm{M}$, capacitor-C) that is only excited by current sources. The incidence matrix of nodes/branches $\mathbf{A}$, the branch currents $\mathbf{i}_{B}$, and nodal voltages $\mathbf{v}_{N}$ can be written as follows:

$$
\begin{aligned}
\mathbf{A}=\left[\begin{array}{llll}
\mathbf{A}_{R} & \mathbf{A}_{C} & \mathbf{A}_{L} & \mathbf{A}_{I}
\end{array}\right] ; & \mathbf{i}_{B}=\left[\begin{array}{llll}
\mathbf{i}_{R} & \mathbf{i}_{C} & \mathbf{i}_{L} & \mathbf{i}_{I}
\end{array}\right]^{T} \\
\mathbf{v}_{N} & =\left[\begin{array}{llll}
\mathbf{v}_{R} & \mathbf{v}_{C} & \mathbf{v}_{L} & \mathbf{v}_{I}
\end{array}\right]^{T}
\end{aligned}
$$

where the subscripts $R, L, M, C$, and $I$ are associated with the branches containing resistors, the partial and mutual inductances (calculated by PEEC integral method), the external capacitors, and the external current sources. The state equations of electrical circuits provide:

$$
\begin{aligned}
\mathbf{i}_{I} & =-\mathbf{I}_{t}(t) ; \mathbf{i}_{R}=\mathbf{R}_{\mathrm{EXT}}{ }^{-1} \cdot \mathbf{v}_{R} ; \mathbf{i}_{C}=\mathbf{C}_{\mathrm{EXT}} \frac{d}{d t} \mathbf{v}_{C} ; \mathbf{v}_{L} \\
& =\mathbf{L}_{\mathrm{EXT}} \frac{d}{d t} \mathbf{i}_{L}
\end{aligned}
$$

where $\mathbf{I}_{t}(t)$ is the vector of current sources, $\mathbf{R}_{\mathrm{EXT}}$ and $\mathbf{C}_{\mathrm{EXT}}$ are diagonal matrices, and $\mathbf{L}_{\mathrm{EXT}}$ is an inductance matrix from external circuit. For full matrix $\mathbf{L}_{\mathrm{PEEC}}$ (inductive coupling PEEC) and resistance diagonal matrix $\mathbf{R}_{\mathrm{PEEC}}$, we have an equation restricted to the PEEC elements:

$$
\mathbf{v}_{l \text { PEEC }}=\mathbf{R}_{\mathrm{PEEC}} \cdot \mathbf{i}_{l \mathrm{PEEC}}+\mathbf{L}_{\mathrm{PEEC}} \frac{d}{d t} \mathbf{i}_{l \mathrm{PEEC}} \cdot
$$




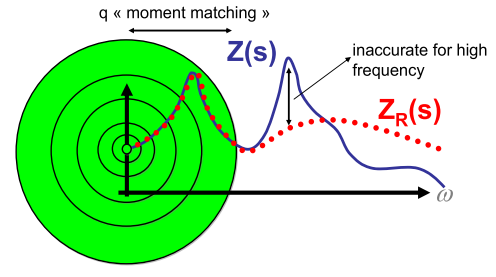

Fig. 1. Taylor expansion around $s=0$.

In order to compute the impedance matrix, the final state space formulations are:

$$
\begin{aligned}
\mathbf{E} \frac{d \mathbf{x}(t)}{d t} & =-\mathbf{G} \cdot \mathbf{x}(t)+\mathbf{B} \cdot \mathbf{u}(t) \\
\mathbf{y}(t) & =\mathbf{B}^{T} \cdot \mathbf{x}(t)
\end{aligned}
$$

where

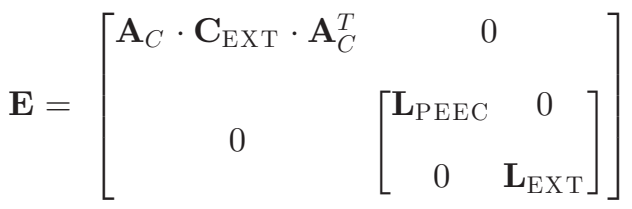

$$
\begin{aligned}
& \mathbf{G}=\left[\begin{array}{cc}
\mathbf{A}_{R} \cdot \mathbf{R}_{\mathrm{EXT}}{ }^{-1} \cdot \mathbf{A}_{R}^{T} & \mathbf{A}_{L} \\
& -\mathbf{A}_{L}^{T}
\end{array}\right] \\
& \mathbf{B}=\left[\begin{array}{c}
\mathbf{A}_{I} \\
0
\end{array}\right] \mathbf{x}=\left[\begin{array}{c}
\mathbf{v}_{N} \\
\mathbf{i}_{L}
\end{array}\right] \mathbf{u}=-\mathbf{i}_{I} .
\end{aligned}
$$

By applying the Laplace transform to (4), we can calculate the matrix $\mathbf{Z}(s)$ or transfer function:

$$
\mathbf{Z}(s)=\mathbf{B}^{T} \cdot(\mathbf{G}+s \cdot \mathbf{E})^{-1} \cdot \mathbf{B} .
$$

\section{Multipoint ExpAnsion MOR COUPLED With FMM}

With an expansion point $s_{0}$, we can rewrite (7) as follows:

$$
\begin{aligned}
\mathbf{Z}(s) & =\mathbf{B}^{T} \cdot\left(\mathbf{G}+s_{0} \cdot \mathbf{E}+\left(s-s_{0}\right) \cdot \mathbf{E}\right)^{-1} \cdot \mathbf{B} \\
& =\mathbf{B}^{T} \cdot\left(\mathbf{I}-\left(s-s_{0}\right) \cdot \mathbf{D}\right)^{-1} \cdot \mathbf{F}
\end{aligned}
$$

and

$$
\mathbf{D}=-\left(\mathbf{G}+s_{0} \cdot \mathbf{E}\right)^{-1} \cdot \mathbf{E} ; \mathbf{F}=\left(\mathbf{G}+s_{0} \cdot \mathbf{E}\right)^{-1} \cdot \mathbf{B} .
$$

In the conventional PRIMA method, only one expansion point $(s=0)$ is used to construct the Krylov subspace created by the method block Arnoldi [10]. This approach is equivalent to a Taylor series expansion of the last (8) at $s_{0}=0$ Fig. 1

$$
\begin{aligned}
K_{m}(\mathbf{D}, \mathbf{F}) & \equiv \operatorname{span}\left\{\mathbf{F}, \mathbf{D} \cdot \mathbf{F}, \mathbf{D}^{2} \cdot \mathbf{F}, \ldots, \mathbf{D}^{q-1} \cdot \mathbf{F}\right\} \\
& =\operatorname{span}\left\{s_{0}\right\}
\end{aligned}
$$

with $q$ order of Taylor series expansion (size of Krylov subspace).
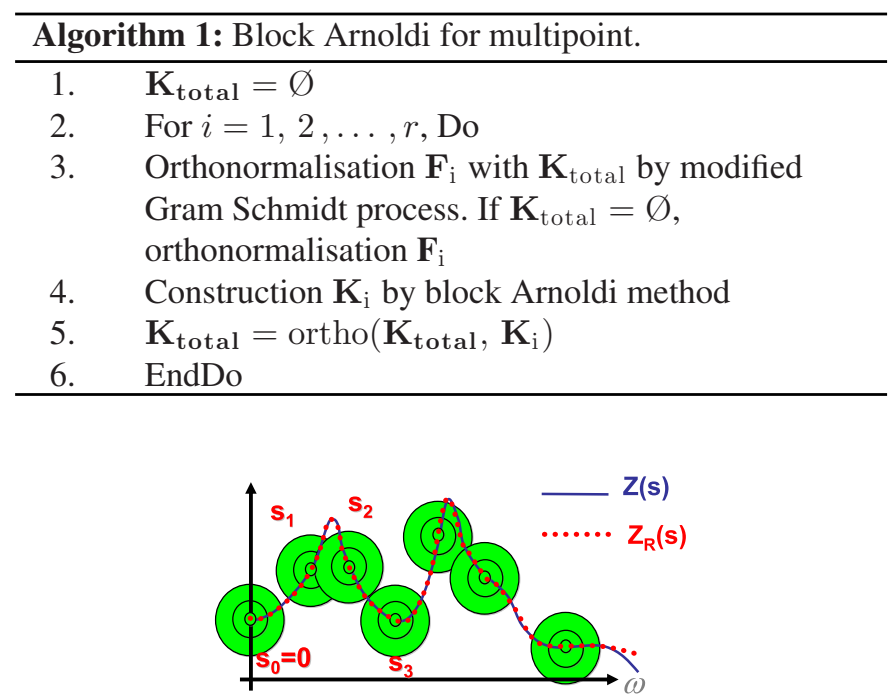

Fig. 2. Taylor expansion around several points $s$.

\section{A. Multipoint Expansion Method}

In this approach, several expansion points are selected for construction of Krylov subspace, the result is then more accurate Fig. 2.

Combination of several subspaces for each expansion point $\mathrm{s}_{j}$ gives us the new Krylov subspace $\mathbf{U}_{m}$ :

$$
\begin{aligned}
\mathbf{U}_{m}= & \operatorname{span}\{\underbrace{\mathbf{F}, \mathbf{D} \cdot \mathbf{F}, \mathbf{D}^{2} \cdot \mathbf{F}, \ldots, \mathbf{D}^{q-1} \cdot \mathbf{F}}_{\text {point } s=0}, \\
& \underbrace{\ldots \mathbf{F}_{j}, \mathbf{D}_{j} \cdot \mathbf{F}_{j}, \mathbf{D}_{j}{ }^{2} \cdot \mathbf{F}_{j}, \ldots, \mathbf{D}_{j}{ }^{q-1} \cdot \mathbf{F}_{j}}_{\text {point } \neq \neq 0}\} \\
= & \operatorname{span}\left\{s_{0}, s_{1}, \ldots, s_{j}\right\}
\end{aligned}
$$

with $\mathbf{D}_{j}=-\left(\mathbf{G}+s_{j} \cdot \mathbf{E}\right)^{-1} \cdot \mathbf{E} ; \mathbf{F}_{j}=\left(\mathbf{G}+s_{j} \cdot \mathbf{E}\right)^{-1} \cdot \mathbf{B}$.

The Krylov subspace, therefore, consists of a first portion corresponding to the first point of expansion and a second portion corresponding to the second point and so on. Let us notice that the vectors constituting this subspace are orthogonal with each other so the blocks representing different points of expansion are orthonormal.

The following algorithm allows us to construct the Krylov subspace in (11) for the expansion point s0, . ., sr [14]. Recall that the Krylov subspace created by an expansion point $s_{\mathrm{j}}$ by starting matrices $\mathbf{D}_{j}$ and $\mathbf{F}_{j}$ is $\mathbf{K}_{j}$. The set of vectors of the Krylov subspace is $\mathbf{K}_{\text {total }}$.

Using Algorithm 1, we have the Krylov subspace $\mathbf{K}_{\text {total }}$ containing several expansion points. The linearly dependent vectors were deleted by deflation process [25]. Finally, we got the Krylov subspace $\mathbf{U}_{m}$. Reduced order matrices obtained with the congruent transformation are then:

$$
\hat{\mathbf{E}}=\mathbf{U}_{m}^{T} \cdot \mathbf{E} \cdot \mathbf{U}_{m} ; \hat{\mathbf{G}}=\mathbf{U}_{m}^{T} \cdot \mathbf{G} \cdot \mathbf{U}_{m} ; \hat{\mathbf{B}}=\mathbf{U}_{m}^{T} \cdot \mathbf{B} .
$$




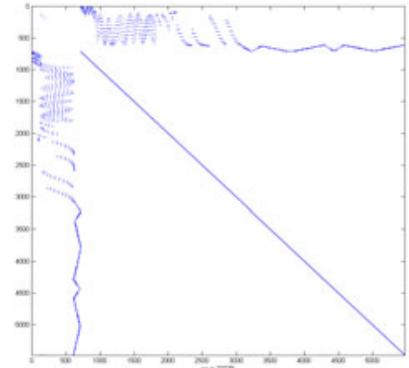

(a)

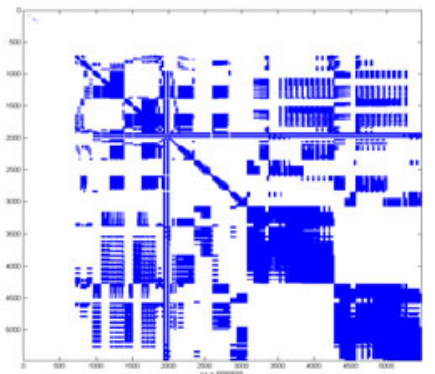

(b)
Fig. 3. Properties of system matrix (a) G matrix (b) E matrix with FMM compressed.

The transfer function $\mathbf{Z}_{R}(s)$ is then reduced:

$$
\mathbf{Z}_{R}(s)=\hat{\mathbf{B}}^{T} \cdot(\hat{\mathbf{G}}+s \cdot \hat{\mathbf{E}})^{-1} \cdot \hat{\mathbf{B}}
$$

All the important properties of PRIMA method like the preservation of passivity and the preservation of moments are conserved. Consequently, the reduced order matrix $\hat{\mathbf{G}}$ is then invertible [25].

For full PEEC matrix, the most consuming time of construction Krylov subspace is to solve the linear system of (9). Without a compression matrix algorithm, the computational cost (matrix-vector product) and the memory cost (for fully dense matrix storage) are at least $O\left(N^{2}\right)(N$ is the size of the matrix) for a good implementation of iterative solver [32]. An AMLFMM compression algorithm is presented in the next section in order to speed-up the computation time and to reduce memory consumption.

\section{B. Fast Multipole Algorithm in Block Arnoldi Process}

The main computational cost of this method is computing matrix-vector product (D. $\mathbf{x}$ in the following) in the block Krylov method to obtain $\mathbf{U}_{m}$.

With the expansion point $\mathbf{S}=0, \mathbf{D} \cdot \mathbf{x}=\mathbf{v}$ is the product made by solving a linear system:

$$
\mathbf{D} \cdot \mathbf{x}=\mathbf{G}^{-1} \cdot \mathbf{E} \cdot \mathbf{x}=\mathbf{v} \Leftrightarrow \mathbf{G} \cdot \mathbf{v}=\mathbf{E} \cdot \mathbf{x} .
$$

The matrix $\mathbf{G}$ is a sparse matrix (which incorporates mainly the resistance); we use a sparse direct LU solver with AMD algorithm in order to conserve the sparse properties of LU decomposition and to enhance the performance [26]. In the case of circuits obtained with PEEC, the matrix $\mathbf{E}$ (especially $\mathbf{L}_{P E E C}$ ) is compressed by an algorithm of type FMM [27] so the matrix-vector product $\mathbf{E}$. x is greatly accelerated from $O\left(N^{2}\right)$ to $O(N \log N)(N$ is the size of matrix $\mathbf{D}$ ). An example of $\mathbf{G}$ matrix is shown in -Fig. 3(a). The white elements in low matrix block in Fig. 3(b) are compressed thanks to FMM. Without FMM, this part is fully dense matrix.

With the expansion point $s \neq 0$, we have a new linear system:

$$
\begin{aligned}
\mathbf{D} \cdot \mathbf{x} & =\left(\mathbf{G}+s_{0} \cdot \mathbf{E}\right)^{-1} \cdot \mathbf{E} \cdot \mathbf{x}=\mathbf{v} \Leftrightarrow\left(\mathbf{G}+s_{0} \cdot \mathbf{E}\right) \cdot \mathbf{v} \\
& =\mathbf{E} \cdot \mathbf{x} .
\end{aligned}
$$

Note that $\left(\mathbf{G}+s_{0} \cdot \mathbf{E}\right)$ is compressed by FMM, so that the system of (15) is no more sparse. To solve (15), we need a solver compatible with FMM (only require product matrix-vector). In this case, iterative solver like GMRES is chosen by its efficiency. However, even if this technique is applied, we may not be able to obtain the solution because of the nonconvergence of GMRES algorithm. Therefore, we need to apply a preconditioning technique in order to ensure the convergence and/or to reduce the number of iterations. To do that, we modify the original system to the new one as (16), where $\mathbf{P}$ is called the preconditioner matrix:

$$
\mathbf{P} \cdot\left(\mathbf{G}+s_{0} \cdot \mathbf{E}\right) \cdot \mathbf{v}=\mathbf{P} \cdot \mathbf{E} \cdot \mathbf{x} .
$$

The new system has a better condition number result in better chance to converge. In theory, the matrix $\mathbf{P}$ should be as similar as possible to $\left(\mathbf{G}+s_{0} \cdot \mathbf{E}\right)^{-1}$ to significantly improve the condition number and speed-up the convergence and the best matrix $\mathbf{P}$ is the matrix $\left(\mathbf{G}+s_{0} \cdot \mathbf{E}\right)^{-1}$. However, to build a preconditioner matrix compatible with the FMM is somewhat different than an explicit matrix solver, because many of the matrix elements are no longer explicitly available. Therefore, the preconditioner has to be built from the near-matrix elements only. We decompose the matrix $\mathbf{E}$ into a near-field component and a far-field one thanks to FMM:

$$
\mathbf{E}=\mathbf{E}_{\text {near }}+\mathbf{E}_{\text {far }} .
$$

The preconditioned matrix $\mathbf{P}$ is built from the total matrix $\mathbf{G}, s_{0}$, and $\mathbf{E}_{\text {near }}$ as (18), where only largest elements in each row of $\mathbf{E}_{\text {near }}$ are contributed to the preconditioned matrix (selected thanks to a coefficient (coeff) range from 0 to 1 ):

$$
\mathbf{P}=\left[\mathbf{G}+s_{0} \cdot \mathbf{E}_{\text {near }}(\text { coeff })\right]^{-1} .
$$

A value in the $i$ th row of the matrix $\mathbf{E}_{\text {near }}$ is retained if this value is bigger than the maximum value of this line (typically diagonal) multiplied by coeff. If coeff equals 0 , the entire matrix $\mathbf{E}_{\text {near }}$ is retained, if the coeff equals 1 , only the diagonal of matrix $\mathbf{E}_{\text {near }}$ is retained. The usage of coeff parameter allows us control the sparsity of matrix $\mathbf{P}$. The sparsity of this matrix is in between matrix $\mathbf{G}$ and $\mathbf{E}_{\text {near }}$ (see Fig. 3) so the direct sparse solver could be used effectively.

In the next section, we will present an algorithm for automated selection of expansion point for a fixed moment matching ( $q$ order) at each point.

\section{AdAptive Multipoint Selection Scheme}

Up to now, IRKA algorithm [19], [20] or CURE framework [21], [22] give the best approximation of optimal $\|\mathrm{H}\|_{2}$-norm reduced model with a fixed $q$-order moment matching, among all reduced systems sharing the same set of poles [20].

In this section, we present the basic idea of this algorithm and then we propose our new algorithm for adaptive selection of interpolation point based on IRKA algorithm.

In theory, for a dynamic system of classic form as (19), the optimal reduced model might be expected to approximate the 
eigenvalues of the original system as (20):

$$
\begin{aligned}
\frac{d \mathbf{x}(t)}{d t} & =-\mathbf{A} \mathbf{x}(t)+\mathbf{B} \mathbf{u}(t) \\
\mathbf{y}(t) & =\mathbf{B}^{T} \mathbf{x}(t) \\
\text { error } & =\|\operatorname{eig}(\mathbf{A})-\operatorname{eig}(\hat{\mathbf{A}})\|<\text { tol. }
\end{aligned}
$$

As in IRKA algorithm for $r$ expansion points, the author propose using $r$ initial shifts randomly distributed within a region containing the mirror image of the numerical range of original system $A$ as starting expansion point and use $r$ eigenvalue of reduced system as a next expansion point in an iterative processes. The algorithm will stop if it reaches the condition as (21) for $r$ values:

$$
\operatorname{error}=\left\|\operatorname{eig}\left(\hat{\mathbf{A}}_{\mathbf{i}+\mathbf{1}}\right)-\operatorname{eig}\left(\hat{\mathbf{A}}_{\mathbf{i}}\right)\right\|<\text { tol. }
$$

It was shown that this algorithm always converges after a few number of step and the resulted model is optimal in $\|\mathrm{H}\|_{2}$-norm sense [19], [28].

However, the value of $r$ (number of expansion point) is not known. The $r$ initial point impact on the iteration process and the choice of these values is important. It could be expensive if we use the eigenvalues of original system. For each refinement step, we need to compute Krylov subspace again and the subspace of previous steps is deleted. At each sample vector of Krylov subspace, we need to solve a large linear system of (16), which is expensive. From the computation point of view, the cost of refinement step is very high.

We should avoid computing the new vectors of Krylov subspace unless it is strictly necessary. For treating the problem of selecting initial points and limiting the number of these points, we propose a novel approach.

To choose a set that covers our space of interest, and because we do not know system frequency response, we select only two initial points $f_{\min }$ and $f_{\max }$ as expansion points. As the properties of Arnoldi process for $q$-order, it is easy to approximate the largest (dominant) eigenvalues of original system within a very small number of iteration. We expect that with these two points we have some information to compute (approximately) all the eigenvalues in the range $\left[f_{\min }, f_{\max }\right]$ thanks to the properties of multipoint expansion.

To do that, we generate the orthonormalized Krylov subspace at two points $\mathbf{U}_{m}=\operatorname{spanf}_{\min }, \mathrm{f}_{\max }$, then we compute the eigenvalues of reduced system:

$$
\lambda_{R}=\operatorname{eig}\left(\hat{\mathbf{G}}^{-1} \cdot \hat{\mathbf{E}}\right) \text {. }
$$

In [29], for classic dynamic system in (19), the poles or peak points will be the imaginary value of eigenvalues:

$$
\text { poles }=\operatorname{imag}[\operatorname{eig}(\mathbf{A})] \text {. }
$$

For our dynamic system of the form as (4), the poles are computed as follows:

$$
\text { poles }=\operatorname{imag}\left[\operatorname{eig}\left(\mathbf{E}^{-1} \cdot \mathbf{G}\right)\right]=\operatorname{imag}\left(\frac{1}{\operatorname{eig}\left(\mathbf{G}^{-1} \cdot \mathbf{E}\right)}\right) \text {. }
$$

Because the eigenvalue computation of original system is expensive, in the same fashion as IRKA algorithm, we compute the poles of reduced system:

$$
\operatorname{poles}_{R}=\operatorname{imag}\left(\frac{1}{\operatorname{eig}\left(\hat{\mathbf{G}}^{-1} \cdot \hat{\mathbf{E}}\right)}\right) .
$$

In [31], the authors indicated that we could find the largest eigenvalues of large scale matrix by Arnoldi method. The more difficulty is to find the smallest eigenvalues, so if we have a reduced system satisfy the condition in (26) for maximal and minimal eigenvalues, our reduced system is nearly optimal as $\|\mathrm{H}\|_{2}$-norm sense

$$
\text { error }=\max \left\{\begin{array}{l}
\| \min (\text { poles })-\min \left(\operatorname{poles}_{R}\right) \|, \\
\| \max (\text { poles })-\max \left(\operatorname{poles}_{R}\right) \|
\end{array}\right\}<\text { tol. }
$$

The eigenvalues of reduced system might be expected to approximate the eigenvalues of original system if the target error tolerance satisfies. In our practical applications, the maximum relative errors are found at the peak points, where the dominant resonance peaks appear, so the choice of expansion points near these peak points will reduce overall error [9]. The only difficulty is how to compute these points precisely.

The IRKA algorithm is suitable for large-scale systems and it has always converged after a small number of steps while using eigenvalues as expansion points [19]. Based on this idea, if we use the points $\min \left(\operatorname{poles}_{R}\right)$ and $\max \left(\operatorname{poles}_{R}\right)$ as expansions points, we will obtain a good approximation of original eigenvalues after few iteration steps. Since poles closest to the expansion point converge fastest, this idea is called thick-restart process in [24].

We propose an original adaptive three points selection scheme to construct reduced order model with automated selection of expansion points.

The main idea of the proposed algorithm is to keep building the projection basis by adding more expansion points in the range of interest until the reduced-order model becomes accurate enough in the neighborhood of the maximum, minimum, and the middle range eigenvalues (three values). The next expansion point is added based on the value of eigenvalue of reduced-order model. The accuracy of the model is assessed by computing error estimators at three points.

In this algorithm at each step, we add the previous subspace in the new subspace because the information from last step remains relevant from cycle to cycle. The reuse of Krylov subspace reduces the computation cost at each refinement step. The middle expansion point $\left(e_{\mathrm{mid}}\right)$ is used not only for testing the precision of our subspace at this point (which may be the maximum error), but also helps to enlarge subspace in order to make the computation of minimal and maximal eigenvalue is more accurate.

If three tolerances of low, high, and middle points are bigger than the target error tolerance (tol), the Algorithm 2 will start by adding more expansion points. First, it adds two expansion points at the step 2(a). In the case the tolerances of the new low 


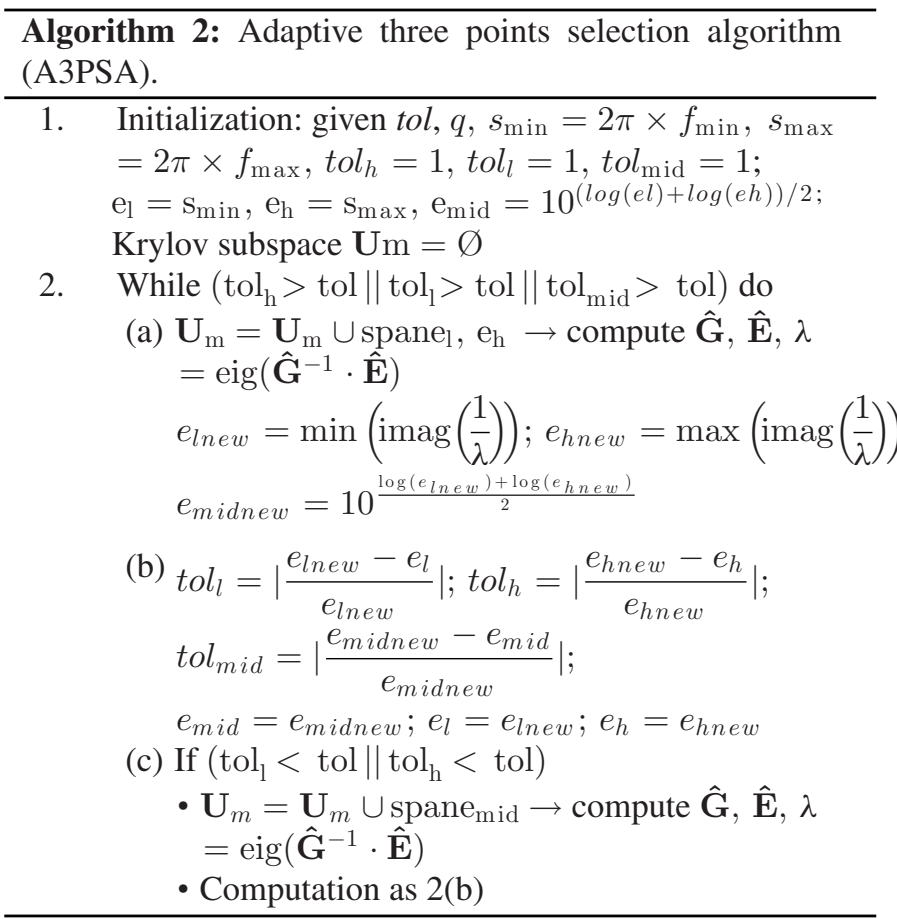

and high points are still bigger than the target error tolerance (tol), the new middle point will be added in the step 2(b).

The expansion point is automatically added at each step: 2 points for minimal and maximal eigenvalues and then 1 middle point if it is necessary. If the last three expansion points converge within the target error tolerance, the algorithm will stop and we have a nearly optimal reduced model in the sense of $\|\mathrm{H}\|_{2}$ minimization. As in [24], this technique makes a smaller reduced model as PRIMA in the same accuracy.

As in [11], the two frequencies $\left[f_{\min }, f_{\max }\right]$ have been chosen for initial expansion points. Ideally, the optimal choice of new expansion point would be the point that causes the largest error but the authors in [11] point out that the choice of new expansion point as the midpoint of others two old expansion points in linear scale cannot warranty the largest errors at this point. In our experience in PEEC modeling, the next expansion point or midpoint should be chosen in logarithmic scale to minimize large error overall. The approach in [11] presents an adaptive scheme of choosing moments but many computation efforts are needed to compute the original model at new expansion point. In our case, the usage of compression algorithm (FMM) cause the exact value of original model even does not exist. In order to reduce the computation time and because of matrix compression property (FMM), in A3PSA algorithm, we use eigenvalues as the stopping criterion and error estimator.

Because of the properties of Krylov subspace and Taylor expansion, it is probable that all the eigenvalues in this range are converged within a target tolerance. This algorithm has the same property of IRKA such as: it converges fast after a few steps so the number of expansion points is small. The number of expansion point depends on the size of q-order. We will discuss about this value in the result section.

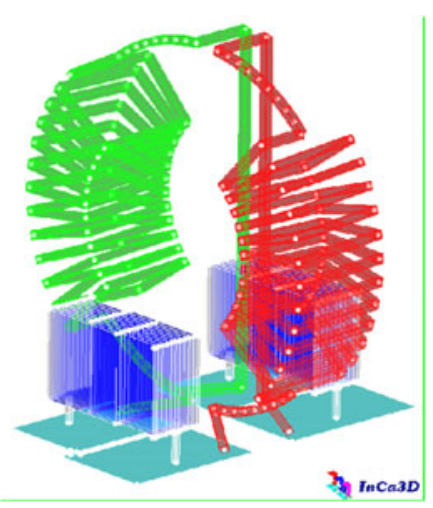

Fig. 4. EMC filter structure in InCa3D software.
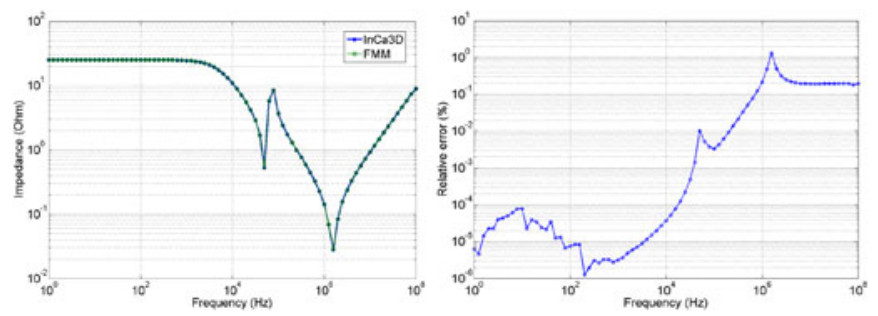

Fig. 5. Comparison of impedances calculated by InCa3D without FMM and FMM version (left) and relative error (right).

We employ real expansion point $s_{0}$ for two reasons. First, the matrices in (15), $\mathbf{E}$ and $\mathbf{G}$, to which the Krylov subspace method is applied, are real, thus avoiding the usage of complex arithmetic, which is four times as costly as real arithmetic. Second, when passive reduced-order models are constructed via the projection approach described in Section III-A, the projection matrix $\mathbf{U}_{m}$ needs to be real in order to obtain real reducedorder matrices (12). On the other hand, the usage of complex expansion points $s_{0}$ typically results in a significantly smaller state-space dimension of the reduced-order models, since $s_{0}$ can be placed closer to the frequency range of interest than any real $s_{0}[25]$.

In the next section, we will present the result for our test problem.

\section{NUMERICAL RESUlTS}

We modeled an EMC filter in the software InCa3D [30] (see Fig. 4) to determine the equivalent impedance.

The state space equation of PEEC discretization gives us an RLMC circuit consisting of 711 nodes, 4767 resistors, five capacitors, 4765 inductors, $4762 \times 4762$ inductive couplings. The frequency range of interest is from $1 \mathrm{~Hz}$ to $100 \mathrm{MHz}$.

The equivalent impedance is calculated by the InCa3D software and with the compression algorithm AMLFMM. Two results are compared in the Fig. 5, the maximal error relative is $1.3 \%$.

In comparison in time and memory of computation with the InCa3D version without FMM, we have the Table I. Let us notice that we use a server with 16-GB memory and four processors Intel Xeon@3.0 GHz for InCa3D version (direct solver) while 
TABLE I

TIME FOR IMPEDANCE COMPUTATION

\begin{tabular}{lcc}
\hline \hline & InCa3D & AMLFMM \\
\hline Time(s) & 22601 & 3500 \\
Memory (MB) & 4900 & 900 \\
\hline \hline
\end{tabular}

TABLE II

Q-ORDER AND EXPANSION POINTS

\begin{tabular}{|c|c|c|c|}
\hline$q$ & MOR size & Time (s) & Expansion points (frequency) \\
\hline 10 & 32 & 491 & $\left(f_{\min }=1, f_{\max }=10^{8}\right), f_{1}=2.36 \times 10^{6}$ \\
\hline 8 & 26 & 411 & $\left(f_{\min }=1, f_{\max }=10^{8}\right), f_{1}=2.37 \times 10^{6}$ \\
\hline 7 & 39 & 609 & $\begin{array}{c}\left(f_{\mathrm{min}}=1, f_{\mathrm{max}}=10^{8}\right), f_{1}=2.57 \times 10^{6},\left(f_{2}=\right. \\
\left.\quad 7.03 \times 10^{4}, f_{3}=7.96 \times 10^{7}\right)\end{array}$ \\
\hline 5 & 29 & 465 & $\begin{array}{c}\left(f_{\min }=1, f_{\max }=10^{8}\right), f_{1}=2.34 \times 10^{6},\left(f_{2}=\right. \\
\left.\quad 7.00 \times 10^{4}, f_{3}=7.91 \times 10^{7}\right)\end{array}$ \\
\hline 4 & 24 & 398 & $\begin{array}{c}\left(f_{\mathrm{min}}=1, f_{\mathrm{max}}=10^{8}\right), f_{1}=2.06 \times 10^{6},\left(f_{2}=\right. \\
\left.7.06 \times 10^{4}, f_{3}=7.94 \times 10^{7}\right)\end{array}$ \\
\hline 3 & 35 & 582 & $\begin{array}{c}\left(f_{\min }=1, f_{\max }=10^{8}\right), f_{1}=4.309 \times 10^{5},\left(f_{2}=\right. \\
\left.7.02 \times 10^{4}, f_{3}=7.16 \times 10^{7}\right), f_{4}= \\
2.28 \times 10^{6},\left(f_{5}=7.03 \times 10^{4}, f_{6}=\right. \\
\left.\quad 8.08 \times 10^{7}\right), f_{7}=2.35 \times 10^{6}\end{array}$ \\
\hline
\end{tabular}

our reduction method with FMM was computed on Intel Core 2 Duo @2.66 Ghz with 2-GB memory. Matrix-vector products were computed by a parallel version of FMM developed in Java. Despite of using FMM, the computation time for this range is still too high (see Table I). The memories used is reduced by 5.4 times from $4900 \mathrm{MB}$ (non-FMM) to $900 \mathrm{MB}$ (with FMM), the runtime decreased from $22601 \mathrm{~s}$ (direct solver LU) to $3500 \mathrm{~s}$ (iterative solver GMRES).

To eliminate the error caused by AMLFMM, we take the result by using compression algorithm as a new reference to compare with our algorithm A3PSA (see Algorithm 2). The accuracy of GMRES will be chosen at 10-12 for resolution of linear systems with point expansion $s_{0} \neq 0$. We chose the coeff equals 0.05 to sparse the matrix $\mathbf{E}_{\text {near }}$.

We run our algorithm with different number of moment matching ( $q$-order) at each expansion point, the tolerance $1 \%$ is chosen. The results later (see Table II) show the size of reduced model, the expansion points created for each Krylov subspace generated, the runtime for generating reduced model.

Let us notice that the bigger value of $\mathrm{q}$ is used, the smaller number expansion point is obtained. For example, $\mathrm{q}=10$, $\mathrm{q}=8$, we need three expansion points. But $\mathrm{q}=5$, we need five points and with $\mathrm{q}=3$, nine points are needed. In Table II, we can see the strategy of adding two points (in parenthesis) or one point. The peak points are well calculated. For all the result obtained, the maximal error is less than the fixed tolerance $1 \%$. The highest computation time is $609 \mathrm{~s}$ at $\mathrm{q}=7$, it reduces with another size of expansion point, which is smaller than $3500 \mathrm{~s}$ as classical computation. Our approach with high accuracy can save not only the time of computation but also the memory requirement.

In the case of different tolerance (no tol, tol $=1 \mathrm{E}-2$ and tol $=1 \mathrm{E}-4)$ and fixed $q=8$, we obtained Table III and Fig. 6 .
TABLE III

DIFFERENT TOLERANCE AND EXPANSION POINTS

\begin{tabular}{lccc}
\hline \hline Tol & MOR size & Time (s) & Expansion points (frequency) \\
\hline- & 17 & 235 & $\left(f_{\min }=1, f_{\max }=10^{8}\right)$ \\
$1 \mathrm{E}-2$ & 26 & 411 & $\left(f_{\min }=1, f_{\max }=10^{8}\right), f_{1}=2.37 \times 10^{6}$ \\
$1 \mathrm{E}-4$ & 53 & 841 & $\left(f_{\min }=1, f_{\max }=10^{8}\right), f_{1}=2.57 \times 10^{6},\left(f_{2}=\right.$ \\
& & & $\left.7.03 \times 10^{4}, f_{3}=7.97 \times 10^{7}\right), f_{4}=2.37 \times 10^{6}$ \\
\hline \hline
\end{tabular}

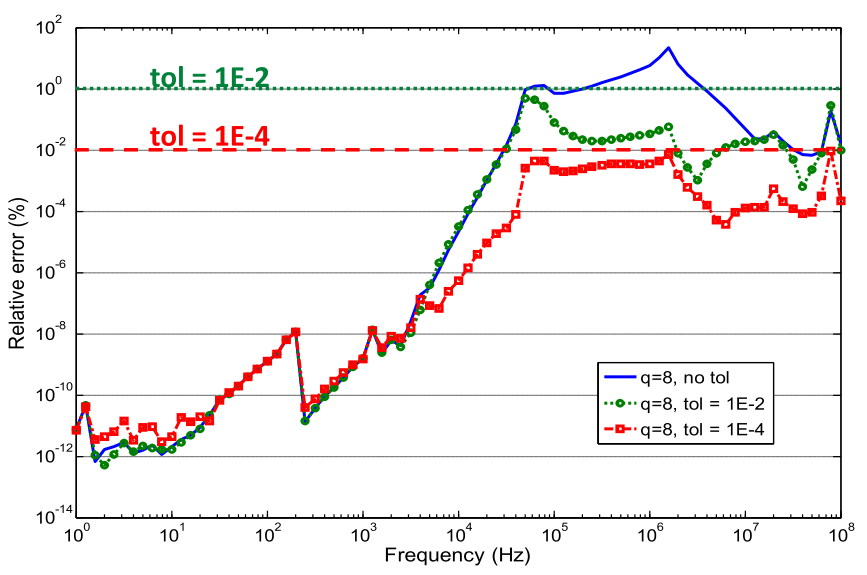

Fig. 6. Relative error of different tolerance, $q=8$.

If no tolerance is chosen, our algorithm use only two expansion points $\left(f_{\min }\right.$ and $\left.f_{\max }\right)$, after $235 \mathrm{~s}$ of computation. If tol equals 1E-4, the A3PSA algorithm stops at six expansion points. It takes $841 \mathrm{~s}$ to obtain MOR size of 53 .

At this value of $q=8$, our algorithm is fast converged after a few steps and the resulted model is accuracy as fixed tolerance of choice. In general, our algorithm can generate rapidly a nearly optimal ROM for a given q-order. In comparison with FMM version, we reduce the runtime from 3500 to $411 \mathrm{~s}$, while using the same amount of memory (900 MB).

\section{CONCLUSION}

In this paper, we have proposed a multipoint rational approximation based on IRKA method for inductive PEEC large circuits compressed with the FMM in the frequency domain. An original algorithm for selecting expansion point automatically is also introduced. The size of reduced order model is nearly optimal for a fixed $q$-order. The reduced-order model is more accurate than one expansion point in PRIMA method for a wide spectrum of frequency. There are still some open problems like the adaptive choice of moment matching number or the choice of complex expansion points, which we are working on it. Regarding the PEEC formulation, one of perspective research is to introduce capacitive and delayed effects [14] in our approach to extend application domain.

To reuse themodel in the time-domain circuit simulation context, a method based on RLMCSYN approaches [5] is needed. The algorithm enables the preservation of PEEC RLM blocks structure and input and output incidence matrices. The final synthesized circuit can then be exported to a SPICE-like solver for the simulation in time domain [5]. 


\section{REFERENCES}

[1] A. E. Ruehli, "Equivalent circuit models for three dimensional multiconductor systems," IEEE Trans. Microw. Theory Techn., vol. MTT-22, no. 3 , pp. 216-221, Mar. 1974.

[2] V. Ardon et al., "EMC modeling of an industrial variable speed drive with an adapted PEEC method," IEEE Trans. Mag., vol. 46, no 8, pp. 2892 2898, Aug. 2010.

[3] T. Le-Duc, O. Chadebec, J.-M. Guichon, G. Meunier, and Y. Lembeey, "Coupling between PEEC and magnetic moment method," COMPEL: The Int. J. Comput. Math. Electr. Electron. Eng., vol. 32, no. 1, pp. 383-395, 2013.

[4] F. Yang, X. Zeng, Y. Su, and D. Zhou, "RLC equivalent circuit synthesis method for structure-preserved reduced-order model of interconnect in VLSI," Commun. Comput. Phys., vol. 3, no. 2, pp. 376-396, 2008.

[5] T.-S. Nguyen, T. Le Duc, S. T. Tran, J.-M. Guichon, and O. Chadebec, "Circuit realization method for reduced order inductive PEEC modeling circuits," COMPEL: The Int. J. Comput. Math. Electr. Electron. Eng., vol. 35, no. 3, pp. 1203-1217, 2016.

[6] A. C. Antoulas, D. C. Sorensen, and S. Gugercin, "A survey of model reduction methods for large-scale systems," Contemp. Math., vol. 280, pp. 193-220, Oct. 2001

[7] T. Wolf, H. Panzer, and B. Lohmann, "Gramian-based error bound in model reduction by Krylov subspace methods," in Proc. 18th IFAC World Congr., Milano, Italy, 2011, pp. 3587-3592.

[8] M. Kamon, F. Wang, and J. White, "Generating nearly optimally compact models from Krylov-subspace based reduced-order models," IEEE Trans. Circuits Syst. II, Analog Digit. Signal Process., vol. 47, no. 4, pp. 239-248, Apr. 2000

[9] E. Grimme, K. Gallivan, and P. Van Dooren, "A rational Lanczos algorithm for model reduction II: Interpolation point selection," Numerical Algorithms, vol. 12, pp. 33-63, 1998.

[10] A. Odabasioglu, M. Celik, and L. Pileggi, "PRIMA: Passive reducedorder interconnect macromodeling algorithm," in Proc. Int. Conf. Comput. Aided-Design, San Jose, CA, USA, Nov. 1997, pp. 58-65.

[11] L. Feng, J. G. Korvink, and P. Benner, "A fully adaptive scheme for model order reduction based on moment-matching," IEEE Trans. Compon., Packag. Manuf. Technol., vol. 5, no. 12, pp. 1872-1884, Nov. 2015.

[12] J. F. Villena and L. M. Silveira, "ARMS-automatic residue-minimization based sampling for multi-point modeling techniques," in Proc. 46th ACM/IEEE Des. Autom. Conf., 2009, pp. 951-956.

[13] L. M. Silveira and J. R. Phillips, "Resampling plans for sample point selection in multipoint model-order reduction," IEEE Trans. Comput.Aided Des. Integr. Circuits Syst., vol. 25, no. 12, pp. 2775-2783, Dec. 2006.

[14] F. Ferranti et al., "Multipoint full-wave model order reduction for delayed PEEC models with large delays," IEEE Trans. Electromagn. Compat., vol. 53, no. 4, pp. 959-967, Nov. 2011.

[15] E. Rasekh and A. Dounavis, "Multiorder arnoldi approach for model order reduction of PEEC models with retardation," IEEE Trans. Compon., Packag. Manuf. Technol., vol. 2, no. 10, pp. 1629-1636, Jul. 2012.

[16] E. Chiprout and M. S. Nakhla, "Analysis of interconnect networks using complex frequency hopping (CFH)," IEEE Trans. Comput.-Aided Design Integr. Circuits Syst., vol. 14, no. 2, pp. 186-200, Feb. 1995.

[17] R. Achar and M. S. Nakhla, "Simulation of high-speed interconnects," Proc. IEEE, vol. 89, no. 5, pp. 693-728, May 2001.

[18] E. J. Grimme, "Krylov projection methods for model reduction," Ph.D. dissertation, Univ. Illinois, Champaign, IL, USA, 1997.

[19] S. Gugercin, C. Beattie, and A. C. Antoulas, "Rational Krylov methods for optimal H2 model reduction," ICAM tech. rep., 2006.

[20] S. Gugercin, A. C. Antoulas, and C. Beattie, "H2 model reduction for large-scale linear dynamical systems," SIAM J. Matrix Anal. Appl., vol. 30, no. 2, pp. 609-638, 2008

[21] H. K. F. Panzer, "Model order reduction by Krylov subspace methods with global error bounds and automatic choice of parameters," Ph.D. dissertation, Universität München, München, Germany, 2014.

[22] T. Wolf, "H2 pseudo-optimal model order reduction," $\mathrm{Ph} . \mathrm{D}$. dissertation, , Universität München, München, Germany, 2014.

[23] M. Rewieński, Lamecki, and M. Mrozowski, "Greedy multipoint modelorder reduction technique for fast computation of scattering parameters of electromagnetic systems," IEEE Trans. Microw. Theory Techn., vol. 64, no. 6, pp. 1681-1693, Jun. 2016.

[24] R. W. Freund, "Recent advances in structure-preserving model order reduction," in Simulation and Verification of Electronic and Biological Systems, P. Li, L. M. Silveira, and P. Feldmann, Eds. Dordrecht, The Nether-
lands/Heidelberg, Germany/London, U.K./New York, NY, USA: Springer, 2011, pp. 43-70.

[25] R. W. Freund, "SPRIM: Structure-preserving reduced-order interconnect macromodeling," in Proc. IEEE/ACM Int. Conf. Comput.-Aided Des., 2004, pp. 80-87.

[26] T. A. Davis, Direct Methods for Sparse Linear Systems. Philadelphia, PA, USA: SIAM, 2006.

[27] A. Jazzar et al., "Modélisation électromagnétique des grands systèmes: l'apport des méthodes intégrales dans l'étude du foudroiement des avions,' Eur. J. Elect. Eng., vol. 16, no. 1, pp. 65-86, 2013.

[28] G. Flagg, C. Beattie, and S. Gugercin, "Convergence of the iterative rational Krylov algorithm,” Syst. Control Lett., vol. 61, no. 6, pp. 688-691, 2012.

[29] P. Kundur, Power System Stability and Control. New York, NY, USA: McGraw-Hill, 1994.

[30] (2015). [Online] InCa3D Software Available at: www.cedrat.com

[31] E. Mark, "The Arnoldi eigenvalue iteration with exact shifts can fail," SIAM J. Matrix Anal. Appl., vol. 31, no. 1, pp. 1-10, 2009.

[32] Y. Saad and M. H. Schultz, "GMRES: A generalized minimal residual algorithm for solving nonsymmetric linear systems," SIAM J. Sci. Statist. Comput., vol. 7, no. 3, pp. 856-869, 1986.

[33] S. Clenet, T. Henneron, and N. Ida, "Reduction of a finite-element parametric model using adaptive POD methods - application to uncertainty quantification," IEEE Trans. Magn., vol. 52, no. 3, pp. 1-4, Mar. 2016.

[34] Y. Sato and H. Igarashi, "Generation of equivalent circuit from finiteelement model using model order reduction," IEEE Trans. Magn., vol. 52, no. 3, pp. 1-4, Mar. 2016.

[35] F. Ferranti, et al. "Interpolation-based parameterized model order reduction of delayed systems." IEEE Trans. Microw. Theory Tech. vol. 60, no. 3, pp. 431-440, Mar. 2012.

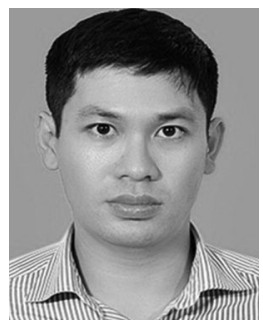

Trung-Son Nguyen received the M.Sc. and Ph.D. degrees in electrical engineering from Grenoble Institute of Technology, Grenoble, France, in 2009 and 2012, respectively.

He held Postdoctoral position with Grenoble Electrical Engineering Laboratory from 2012 to 2013. In 2015, he moved to Vietnam Fire and Rescue Police Department, Vietnam, where he is currently a Researcher of Science - Technology Research \& Apparatus and Equipment Inspection Division. His current research interests include model order reduction, circuit realization method, and development of numerical tools for modeling of electromagnetic phenomena (integral methods).

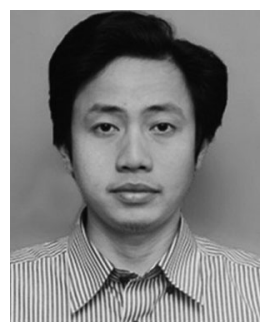

Tung Le Duc was born in 1984, Thanhhoa, Vietnam. He received the Engineer's degree in electrical engineering from Hanoi University of Science and Technology, Hanoi, Vietnam, in 2007, and the M.Sc. and $\mathrm{Ph} . \mathrm{D}$. degrees in electrical engineering from Grenoble Institute of Technology, Grenoble, France, in 2008 and 2011, respectively.

He held Postdoctoral position with Grenoble Electrical Engineering Laboratory, Grenoble, France, from 2011 to 2012. He is currently a LecturerResearcher of electrical engineering at Hanoi University of Science and Technology. He has been a Visiting Scientist at the Technical University of Catalonia, Barcelona, Spain, in 2014. His research interests are in electromagnetic modeling, power systems computations, optimizations, and grid-connected renewable energy systems. 


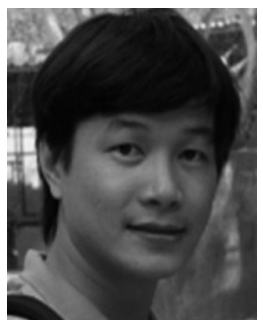

Thanh-Son Tran received the Engineer's degree in electrical engineering from Hanoi University of Science and Technology, Hanoi, Vietnam, in 2004, the M.Sc. degree in electrical engineering from Grenoble Institute of Technology, Hanoi, in 2005, and the $\mathrm{Ph} . \mathrm{D}$. degree in electrical engineering from Joseph Fourier University, Grenoble, France, in 2008.

$\mathrm{He}$ was a Postdoctoral Researcher in Grenoble Institute of Technology Enterprise from 2009 to 2010. He is currently the Dean of Electrical Engineering Faculty, Electric Power University, Hanoi. His research interests are power systems computations, optimizations, electromagnetic modeling, and numerical methods.

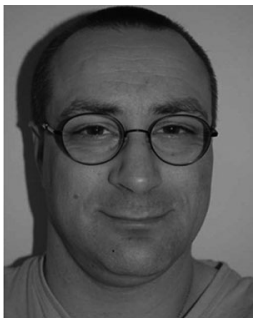

Jean-Michel Guichon received the Diploma degree in electrical engineering and the $\mathrm{Ph} . \mathrm{D}$. degree from Grenoble Institute of Technology, Grenoble, France, in 1998 and 2001, respectively.

$\mathrm{He}$ is an Associate Professor at the University Grenoble Alpes, Grenoble, France. He is has been with the Grenoble Electrical Engineering Laboratory, since 2003, working in the field of electromagnetic modelization for power electronics. He is focused in the development of numerical tools to compute the electromagnetic field and the electrical behavior in low frequency (without propagation).

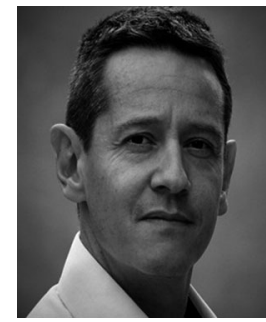

Olivier Chadebec was born in 1973, Sens, France. $\mathrm{He}$ received the Diploma in electrical engineering and the Ph.D. degrees from the Grenoble Institute of Technology, Grenoble, France, in 1997 and 2001, respectively.

$\mathrm{He}$ is currently a CNRS Senior Researcher (directeur de recherche $C N R S$ ) and leads the "Models, Methods and Methodologies Applied to Electrical Engineering" research group of G2ELab, University of Grenoble Alpes, Grenoble. He has been a Visiting Scientist at the Technology Centre of the Federal University of Santa Catarina, Brasil, in 2012-2013. His research activities focus on computational electromagnetism (finite element and integral methods), equivalent magnetic sources identification by solving inverse problems and low magnetic fields measurements. He has coauthored more than 140 papers published in international journals and conference proceedings.

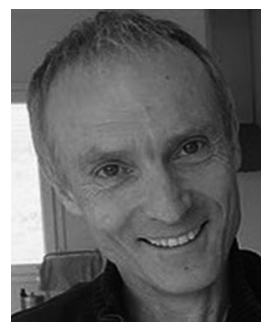

Gérard Meunier received the Dipl.-Ing. in electrical engineering and the Ph.D. degree from the National Polytechnic Institute of Grenoble (INPG) Grenoble, France, in 1977 and 1981, respectively.

He joined the CNRS and the Power Electrical Engineering Laboratory of Grenoble (G2Elab), Grenoble, in 1982, where he is currently a Senior Researcher. His researches are devoted to numerical modeling of electromagnetic phenomena. In the G2Elab, he was successively responsible of the "Modeling and CAD team" from 1990 to 1998 and an Associate Director from 1998 to 2002. He was responsible of the Power Electrical Engineering Doctoral Department from 2002 to 2015 and is currently a Deputy Head of the EEATS Doctoral School of the Communauté Université Grenoble Alpes, Grenoble. 